\title{
Nanotechnology: a revolution in targeted drug delivery
}

\author{
Debaleena Das*, Nivedita Maity, Anuradha H. V.
}

Department of Pharmacology, Ramaiah Medical College, Bangalore, Karnataka, India

Received: 04 October 2017

Revised: 14 October 2017

Accepted: 27 October 2017

\section{*Correspondence to:}

Dr. Debaleena Das,

Email: drdebaleena08@ gmail.com

Copyright: (C) the author(s), publisher and licensee Medip Academy. This is an openaccess article distributed under the terms of the Creative Commons Attribution NonCommercial License, which permits unrestricted noncommercial use, distribution, and reproduction in any medium, provided the original work is properly cited.

\begin{abstract}
Targeted drug delivery is a method of delivering medication in a unique way so that the concentration of the drug at the target site is optimized, burden of the drug to other tissues is reduced and toxicity minimized. There are various novel approaches to deliver drugs to the target sites in the form of nanocapsules, nanocrystals, nanoemulsions, dendrimers, polymeric micelles, nanotubes and monoclonal antibodies. They have the advantages of improved bioavailability, facilitated transport of drugs across difficult barriers to reach the target tissues for a specific action and providing protection to protect the drug against degradation. A number of anticancer drugs like Doxorubicin, Paclitaxel and 5-Fluorouracil have been formulated using nanoparticles. These innovative techniques have helped to overcome the limitations like resistance in the target cells and difficulty in movement across the barriers which are seen in the conventionaldrug delivery system. Apart from being a therapeutic tool, it also has brought revolution in early diagnosis of diseases and gene transfer. The surge of nanotechnology is now being translated into commercialized products. The future is exciting, and the promises are limitless as the application of nanotechnology in medicine will provide remarkable opportunities and newer pers pectives for novel and effective treatment in various diseases.
\end{abstract}

Keywords: Dendrimer, Micelle, Nanotechnology, Targeted drug delivery

\section{INTRODUCTION}

Simplest definition of Nanotechnology could be 'Technology at Nanoscale' ranging from 0.1 to $100 \mathrm{~nm}$ $\left(1 \mathrm{~mm}=10^{-9} \mathrm{~m}\right)$. A more specific definition by the US National Nanotechnology Initiative is "Nanotechnology is concerned with materials and systems whose structures and components exhibit novel and significantly improved physical, chemical and biological properties, phenomena and processes due to their nanoscale size". ${ }^{1}$

It is also defined as the understanding, control and manipulation of matter on the order of nanometers to create fundamentally new materials with innovative properties, precision and functions. Molecular nanotechnology holds a great promise for breakthroughs in the manufacturing industry, electronics, medicine, biotechnology, information technology and national security. ${ }^{2}$

The present review outlines the tremendous potential and recent advances of nanotechnology in the arena of targeted drug delivery system, the major goal of which is to deliver pharmacologically active moiety to pre-identified or sitespecified targets in therapeutic concentration while restricting its access to non-target cells. ${ }^{3,4}$ Benefits of nano drug delivery systemare manifold in a manner that the biodegradable drugs are protected against enzymatic degradation, their toxic effects are minimized, while the therapeutic efficacy are maximized. ${ }^{5}$ 


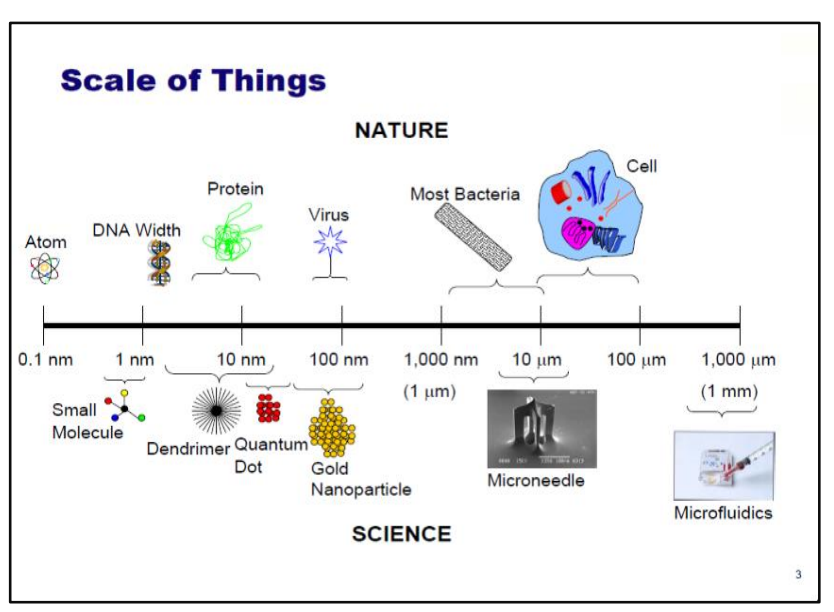

Figure 1: Dimension of nanomaterials. ${ }^{6}$

\section{HISTORY}

The earliest mention of nanotechnology was made in a speech given by Richard Feynman in 1959 titled "There's Plenty of Room at the Bottom."7 'Nanotechnology' was first coined by a Japanese scientist Norio Taniguchi in the year 1974. An eminent American engineer K. Eric Drexler was the one who brought revolution in the field of molecular nanotechnology. ${ }^{8}$ In the late 1990s and early 2000s almost all industrialized nations took nanotechnology initiatives, ${ }^{9}$ leading to a worldwide increase in research which further led to manufacturing of molecular nanosystems. Since then nanotechnology has progressed to bring about revolution in early disease detection and treatment.

\section{NEED OF NANOTECHNOLOGY IN MEDICINE}

Emerging nanotechnologies are revolutionizing the field of 'Theranostics' by being strategically implemented in drug development and delivery systems that can expand drug market exponentially. ${ }^{10}$ Its applications have paved the pathway of an emerging discipline, 'Nanomedicine', which is the study of nanomaterials involved in the betterment of diagnosis, control, prevention and treatment as well.

Many a times full scale development of a potential drug candidate is rejected because of safety or efficacy issues related to degradability by gastric acid, poor solubility, poor permeability and poor bioavailability. Nano based drug delivery gives stability to the hydrophobic drugs, improves biodistribution and rate of dissolution resulting in improved efficacy. In nano delivery systems, drugs are protected from degradation by an encapsulation of lipid polymer and its dispersement inside a matrix. Additionally, an enhanced permeability and retention effect enable the drugs to accumulate in the desired site like tumour, inflammatory or infectious cells. ${ }^{11}$

The other possible benefits of nanotechnology are controlled drug release with less amount of drug requirement; reduced adverse effects as a consequence of favoured accumulation at target sites and decreased toxicity to non-target cells by using biocompatible nanomaterials. ${ }^{12}$

\section{Components of targeted drug delivery}

- Target: Specific organ or cell group

- Carrier/Marker: Special molecule for transportation of drugs to pre-specified sites with engineered vectors loaded with drugs on or inside them

- Drug: To be delivered

\section{DRUG TARGETING STRATEGIES}

Since Paul Ehrlich, "Father of chemotherapy", conceived the concept of a "Magic bullet", i.e., "A drug that selectively targets the diseased cells but is non-toxic to healthy cells" has a great deal of significance in the treatment of cancer. ${ }^{13}$ Various strategies can be exploited to reach a biological target by the following: $:{ }^{14}$

\section{Active targeting}

In this technique, targeting ligands are attached at the surface of the nanocarrier which transports the drugs to the specific sites based on the modifications made on the surface (Figure 2). ${ }^{15}$

\section{Passive targeting}

Based on the property that certain colloids are taken up by reticuloendothelial system (RES), thereby transports the drugs to liver and spleen by targeting the systemic circulation (Figure 2). ${ }^{16}$

\section{Inverse targeting}

RES uptake of drugs is prevented by saturating it with blank colloidal substrate or macromolecule like dextran. ${ }^{16}$

\section{Dual targeting}

Both the carrier molecule and drug have therapeutic value in this system. ${ }^{16}$

\section{Double targeting}

Temporal (controlling the rate of drug delivery) and spatial (targeting drugs to specific organs tissues) methodologies are combined. ${ }^{16}$

\section{TECHNOLOGIES AND MATERIALS USED IN NANOPARTICLE MANUFACTURING ${ }^{16}$}

\section{Biologic}

In this technology, peptides and lipids are used to generate various nanoparticles. 


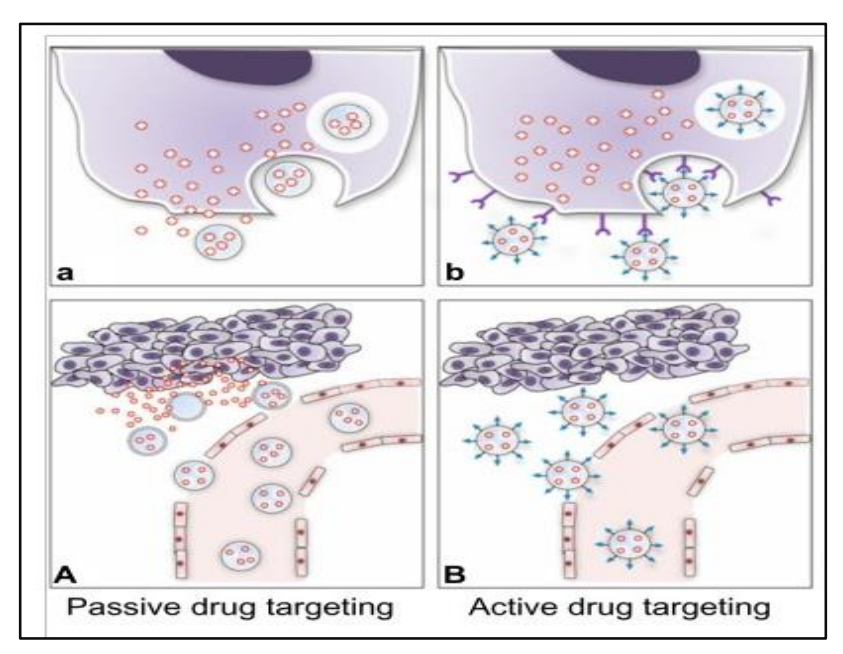

Figure 2: Strategies adopted for drug targeting. ${ }^{17}$

\section{Polymeric}

In the polymeric technology, Poly (glycolic acid), poly (lactic acid), poly (ethylene glycol) and poly(caprolactone) are utilised to yield nanoparticles like vescicles, spheres, micelles and dendrimers.

\section{Silicon based}

Materials like Silicon, $\mathrm{SiO}_{2}$ are used to produce nanoneedles.

\section{Carbon or metal based}

Carbon, palladium, platinum, gold is used as materials to structure nanoshells, nanotubes, fullerenes like nanoparticles.

\section{METHODS USED IN PREPARATION OF NANOPARTICLES ${ }^{12}$}

Various methods are employed to manufacture nanoparticles depending on the raw material intended to be used and on the characteristics of the drug. Few methods of preparations of nanoparticles are as follows:

\section{Nanospheres Preparation}

Nanospheres can be prepared by the following methods:

- In-situ polymerization

- Emulsion-evaporation

- Salting out

\section{Solid-Lipid Nanoparticles (SLN)}

Different methods used to prepare the SLNs are as follows:

- High shearhomogenization

- Hot homogenization

- Cold homogenization

\section{Nanocrystals preparation}

Methods used for preparation of nanocrystals are as follows:

- Milling

- Precipitation

- Homogenization method

\section{APPLICATIONS OF NANOTECHNOLOGY IN THERAPEUTICS}

\section{Cancer therapy}

\section{Dendrimers}

Dendrimers are nano-sized, radially symmetrical molecules with well-defined, spheroid or globular, branched structures radiating from its internal cavity leading to formation of successive generations of shells and have chemical polymer. ${ }^{18}$ It also have low cytotoxicity, high specificity and can be precisely engineered to carry bioactives like drugs, vaccines and genes to specified sites in the space between the core and branches. ${ }^{8}$ Number of generations of the shells, chemical composition of the core, interior branching and surface functionalities determine the size, shape and reactivity. The utilization of dendrimers as drug delivery carriers is highly beneficial due to their highly controllable structure and size. Dendrimers are synthesized generally by divergent or convergent method. Conjugation of drugs to Polyamido amines (PAMAM) dendrimers as drug delivery systems has been translated and found to be efficacious. ${ }^{19}$ For example, 5-fluorouracil (5FU)-PAMAM dendrimer conjugates slowly release $5 \mathrm{FU} .^{20}$

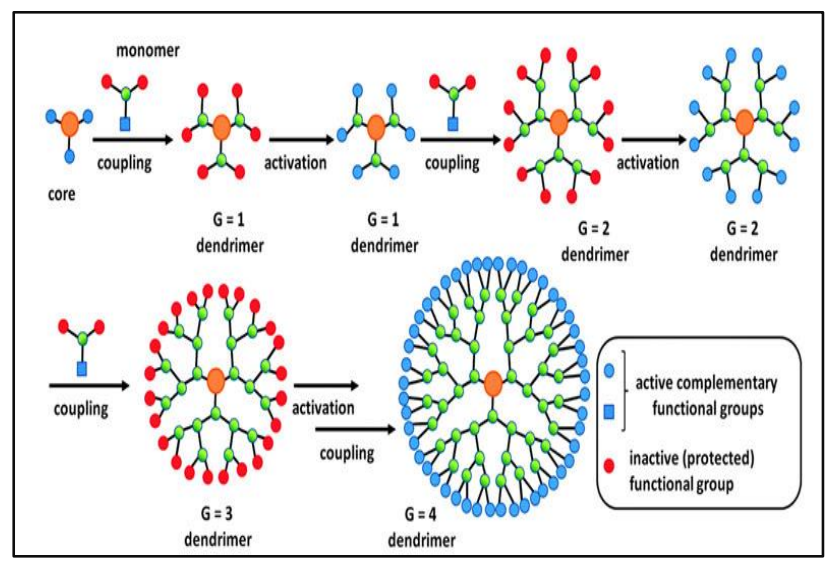

Figure 3: Dendrimers. ${ }^{21}$

Polymeric nanoparticles

Polymeric nanoparticles are biocompatible, nonimmunogenic, non-toxic and biodegradable colloidal carrier having a dimension of $10 \mathrm{~nm}-1 \mu \mathrm{m}$. Polymeric nanoparticles are classified as nanocapsules and nanospheres. ${ }^{8}$ 


\section{i) Nanocapsules}

These are the structures in which the drugs are confined to a cavity surrounded by an unique polymeric membrane whereas in nanospheres drug is dispersed throughout the polymer matrix. $^{22}$ Natural polymers like gelatin, albumin and alginate are used in the preparation of these nanoparticles. Synthetic polymers can also be used e.g. polyesters like polycaprolactone. The concept of the capsule is nothing but to provide a templating core to accomodate the drug particles which are surrounded by multilayered polymeric spheres with light absorbing gold nanoparticles. As sufficient number of capsules accumulate in the malignant cells, a low energy pulse from a near-infrared laser is applied. The infrared energy $\left(100 \mathrm{~mJ} / \mathrm{cm}^{2}\right)$ pulse ruptures the polymer spheres and subsequently helps the nanostructured capsules to release their contents. ${ }^{23}$ Cisplatin and carboplatin can be delivered through nanocarriers. ${ }^{24}$

\section{ii) Nanospheres}

Nanospheres are the spherical particles with the dimension between 10-200 $\mathrm{nm}$ in diameter. Basically the drug is dissolved, entrapped and encapsulated to the matrix of polymer in which the drug is uniformly dispersed. Nanospheres are generally amorphous or crystalline in nature with the ability to protect the drug from enzymatic and chemical degradation. It has controlled release property and site specific targeting by attaching the ligands to the surface of the spheres. One of the drawbacks is that the physicalhandling of nanospheres is difficult in liquids and in dry form. Due to the larger surface area of nanospheres with smaller size, chances of particle aggregation increase and drug loading or the burst release is limited. ${ }^{25}$

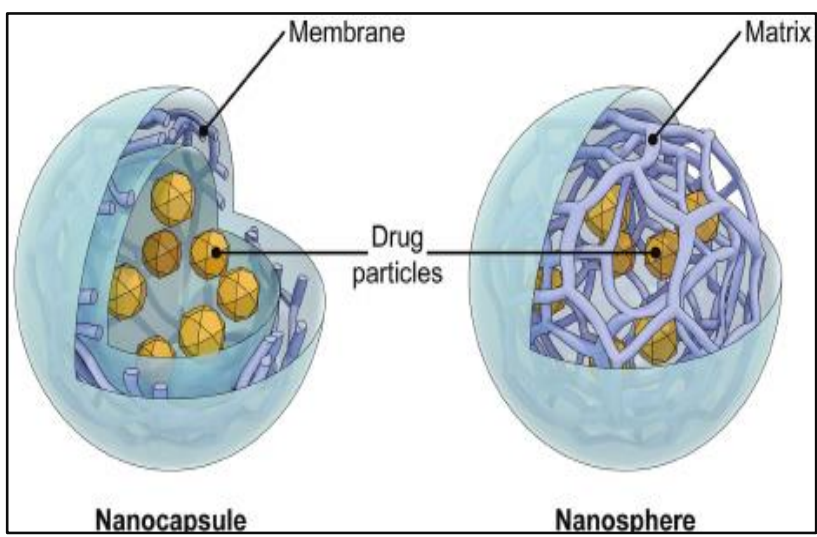

Figure 4: Schematic representation of the structure of a nanocapsule and a nanosphere. ${ }^{26}$

\section{Magnetic nanoparticles}

Magnetic nanoparticles have wide applications in the field of magnetic immunoassays, drug delivery and tissue repair. They are structured particles made up of magnetic materials like superparamagnetic $\mathrm{Fe}_{3} \mathrm{O}_{4}, \mathrm{Fe}_{2} \mathrm{O}_{3}$ and many other ferrites. ${ }^{27}$ Surface functionalized iron oxide based nanoparticles with superior magnetic properties are being intensively investigated to achieve highly efficient carcinogenic cell destruction by inducing hyperthermia. ${ }^{28}$ Nature of the surface coatings determines the kinetics involved with these particles as well as in their separation and purification. Co-precipitation, thermal decomposition and reduction, micelle synthesis is some of the procedures involved in the development of these particles. ${ }^{29}$ A noninvasive targeted therapy for the treatment of solid breast tumours known as 'Targeted Nano Therapeutics System' has been introduced which contains polymer-coated superparamagnetic iron oxide nanoparticles bound to the monoclonal antibodies. This "bioprobe" once injected into the blood, detects and binds the cancer cells with the help of the antibodies attatched. ${ }^{23}$

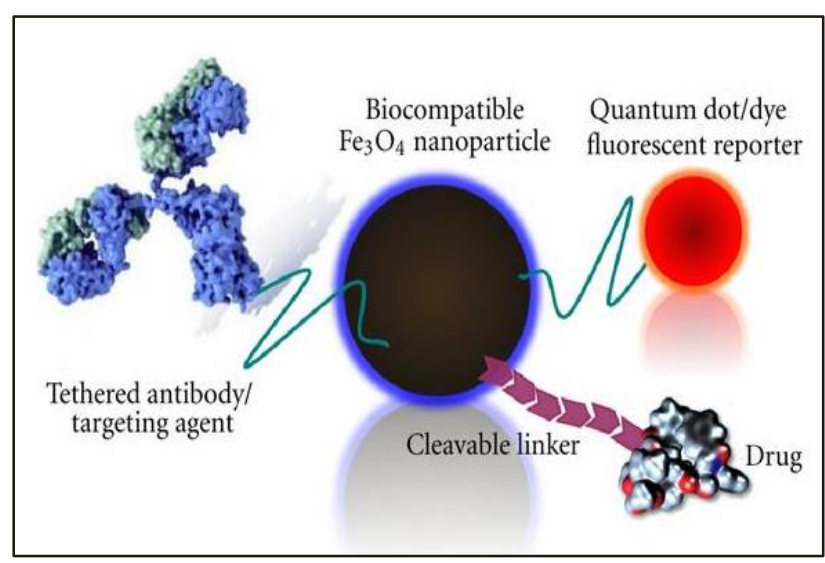

Figure 5: Magnetic nanoparticle. ${ }^{30}$

\section{Quantum dots}

These are tiny crystals consisting of a semi-conductor inner core surrounded by a shell to improve optical properties. ${ }^{31}$ Their properties are due to their physical size which ranges from $10-100 \mathrm{~A}^{0}$ in radius. They emit light when these are stimulated by ultraviolet ray. ${ }^{32}$ By combining different sized quantum dots within a single bead probe can be created which can be tuned to absorb and emit a distinct spectrum of light in the near infrared region by changing their size and composition and specific cells are visible. These latex beads are filled with crystals designed to bind to specific DNA sequences. Low intensity light can be used to penetrate tissues of deepseated tumours. ${ }^{33}$ Their usage in imaging, in-vitro and invivo detection and analys is of biomolecules, immunoassay and DNA hybridization and as non-viral vectors for gene therapy is impressive. Their main function is to label the cells and act as therapeutic tools for cancer.

\section{Liposomes}

Liposomes are spherical, closed, colloidal structures composed of lipid bilayers that surround a central aqueous core. These have shown an ability to improve the pharmacokinetic and pharmacodynamic profiles of the 
as sociated drugs. The phospholipid, ranging between 50$700 \mathrm{~nm}$ in size, is predominantly phosphatidylcholine comprising of a hydrophilic head and two lipophilic tails. ${ }^{34,35}$ The size and shape of the vesicles are not fixed as their preparations are metastable.

\section{i) Small and large nilamellarvescicles}

They can be categorised on the basis of size and the number of lipid bi-layers as small and large unilamellar, which contain a single lipid bi-layer surrounding the aqueous core. ${ }^{27}$ Drug is entrapped or intercalated in the aqueous space into lipid bi-layer of liposome. Diversity in shape, texture, size, flexibility and a variety of surface modification enables them for both active and passive delivery of bioactives. Their application in cancer therapy is now well established. ${ }^{8}$

\section{ii) Multilamellarvesicles}

These liposomal structures consist of several lipid bilayers separated from one another by aqueous spaces. They are heterogeneous in size varied between 100 to $1000 \mathrm{~nm}$. Liposomes are widely used in the field of imaging and drug delivery. Super-paramagnetic liposomes can be good MRI (Magnetic Resonance Imaging) contrast agents. Maghemite particles were introduced into a liposomal vesicle and further functionalized by pegylation following which it was administered intravenously in the mouse and magnetic resonance angiography (MRA) was done for better precision. ${ }^{36}$

Several liposome based anticancer drugs have been approved for the treatment of metastatic breast cancer and kaposi's sarcoma. ${ }^{32}$ These nanocarriers are biodegradable, biocompatible, non-toxic, flexible, non-ionic, can carry both water soluble and lipid soluble drugs and have the flexibility to couple with site-specific ligands to achieve active targeting. However, short half-life, high cost, low solubility, leakage and fusion of encapsulated drug molecules are their biggest disadvantages.${ }^{16,27}$ Liposomal entrapment and delivery of doxorubicin, daunorubicin and vincristine have showed reduced cardiotoxicity. ${ }^{37}$

\section{Photothermal ablation therapy}

In general, thermal ablation therapy deals with hyperthermia as well as thermotherapy. Hyperthermia therapy is based on the fact that tumour cells are more vulnerable to temperature increase than normal tissue cells. Heating to temperature between $41-45^{\circ} \mathrm{C}$ induces reversible damage to cells and tissues while for thermal ablation therapy, higher temperature ranging from $50^{\circ} \mathrm{C}$ to $70^{\circ} \mathrm{C}$ is applied, leading to the destruction of pathologically degenerated cells. In case of successful treatment, generally the tumour disappears, diminishes or regresses.

In the murine model polyethylene glycol-pas sivated goldcoated silica nanoshells are injected interstitially into the core of the tumour where polyethylene glycol is used to enhance specificity. The leaky blood vessels inside the tumours allow the nanoshells to extrude out and accumulate inside tumours.

Later, an infrared light is applied on the skin six hours after the injections for 3-7 minutes. ${ }^{38}$ The gold nanoparticles readily absorb the energy and turn it into heat which induces irreversible cancerous tissue damage. They are an effective alternative to surgery and chemotherapy. ${ }^{23}$ They provide tissue selective targeting and intracellular targeting of mitochondria. ${ }^{38}$

\section{Carbon nanotubes}

Carbon Nanotubes are allotropes of carbon with a nanostructure that possess a length-to-diameter ratio greater than $1,000,000$. They are the members of fullerene structural family, which is a large class of allotropes of carbon and are made up of balls, cages or tubes of carbon atoms. ${ }^{39}$ Cylindrical fullerenes are also called carbon nanotubes having a similar structure of graphite. Their exceptional electrical, chemical and mechanical properties depend on their diameter, length and chirality contributing to their unique surface area, strength and resilience and making them suitable for detection, monitoring and therapy of cancer. ${ }^{40}$ For example, these nanotubes help to identify Deoxyribonucleic acid (DNA) changes associated with cancer and location of the mutations. Carbon nanotubes are classified 41 as multi-walled nanotubes (MWNTs) or single-walled nanotubes (SWNTs). SWNT is a single-rolled graphene and MWNT are nanotubes where multiple layers of graphite are rolled around one another to form a tubular shape. ${ }^{37}$
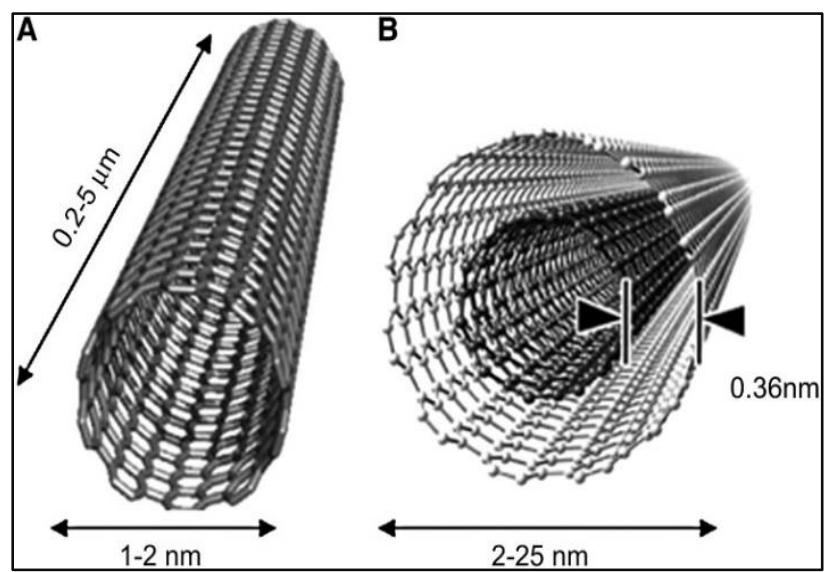

Figure 6: Diagram of (a) single-walled and (b) multiwalled carbon nanotube. ${ }^{40}$

\section{Microsphere}

Microspheres are unique free flowing powders which consist proteins or synthetic polymers and biodegradable in nature having a particle size of less than $200 \mu \mathrm{m}$. They can be prepared from various natural and synthetic materials. ${ }^{42}$ 
Natural sources of microspheres are protein (albumin, gelatin, collagen), carbohydrate (agarose, carrageenan) or chemically modified carbohydrate (polydextran, starch) whereas the synthetic sources of microspheres can be biodegradable (Lactides, Glycolide copolymer) or nonbiodegradable (Polymethyl methacrylate or PMMA). There are various types of microspheres namely magnetic, bioadhesive, radioactive, floating and polymeric. ${ }^{42}$ Different techniques that are used for the preparation of microspheres are single emulsion technique, double emulsion, phase separation coacervation and spray congealing techniques. ${ }^{43} \mathrm{~A}$ favourable pharmacokinetic profile, tissue distribution, cellular concentration, controlled release formulation and reduced adverse effects of the drug contribute to minimizing the dose and improve patient compliance. ${ }^{42}$

\section{Radiotherapy}

Carbon nanotubes, gold particles and dendrimers play a major role in radiotherapy. In boron neutron capture therapy patient is injected with a nonradioactive stable isotope of boron incorporated in a dendrimer that selectively find signature chemical sequences in the cancer cells. The patient is then irradiated with a low-energy neutron beam. The neutrons in the beam interact with the boron which is already placed in the tumour causing the boron atom to break down into an alpha particle and a lithium-7 ion. By this technique, selective delivery of the drug to each cancer cell can be achieved. PAMAM dendrimers containing folic acid conjugates of boronated polyethylene glycol units are designed to bind folate receptors which are expressed abundantly in variety of human tumour cells.
Gold Nanoparticles (AuNPs) appear to be of great interest in the field of radiotherapy due to their good biocompatibility, availability of wide range of sizes and easy surface functionalisation required to target cancer cells.

\section{Infectious diseases}

Although many methods for diagnosing and treating infectious diseases are available presently, there is an essential need for innovative approaches for bacterial destruction. Therapeutic efficacy of many drugs may be well recognized; however, inefficient delivery could result in insufficient therapeutic effect and development of resistance. It is evident that nanoparticles due to their capacity to selectively target and destroy pathogenic bacteria have the capacity to overcome these short comings.

Nanotechnology imparts a much awaited breakthrough in medical approaches to treatment and immunity induction as nano anti-microbial agents and vaccine adjuvants target difficult organisms such as Human Immunodeficiency virus, Salmonella, Tuberculosis and Listeria efficiently. Nanoparticles loaded with the small molecules like proteins, peptides and nucleic acids are protected from hepatic inactivation, enzymatic degradation and rapid clearance in vivo. Additionally, co-delivery of two or more drugs are also possible. Liposomes are used as a carrier of rifampicin in respiratory infection whereas tobramycin is transported in solid lipid nanoparticles in Pseudomonas aeruginosa infection. However, it is also important to keep in mind the possible biological and environmental hazards of nanoparticles as well.

Table 1: Marketed nano-carrier based drugs..$^{32}$

\begin{tabular}{|c|c|c|c|}
\hline Formulation & System & Route & $\begin{array}{l}\text { Observed pharmacokinetics/ pharmacodynamics in } \\
\text { vivo }\end{array}$ \\
\hline \multicolumn{4}{|r|}{ 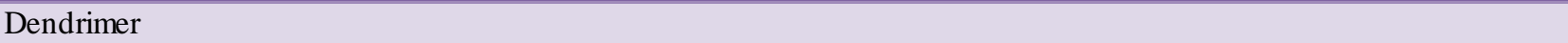 } \\
\hline Doxorubicin & Polylysine dendrimer & IV & enhanced accumulation in tumor tissues \\
\hline Flurbiprofen & Poly(amidoamine) & IV & High distribution and retention in site of inflammation \\
\hline \multicolumn{4}{|c|}{ Engineered nanoparticle } \\
\hline Danazol & Nanocrystals & oral & Improved oral bioavailability \\
\hline \multicolumn{4}{|l|}{ Liposome } \\
\hline Amikacin & Liposome (Phospholipid) & IV & Extended half-life of the drug in vitreous \\
\hline Amphotericin B & Liposome & IV & Increased systemic exposure, decreased RES uptake \\
\hline Doxorubicin & $\begin{array}{l}\text { Liposome, PEGylated } \\
\text { liposome }\end{array}$ & IV & High distribution in neoplastic tissue \\
\hline \multicolumn{4}{|c|}{ Solid lipid nanoparticles } \\
\hline Azidothymidine & Solid lipid nanoparticles & IV & Enhanced permeability and retention to brain \\
\hline $\begin{array}{l}\text { Diclofenac } \\
\text { sodium }\end{array}$ & Solid-in-oil nano suspensions & Dermal & Increased percutaneous absorption \\
\hline \multicolumn{4}{|l|}{ Micelle } \\
\hline Camptothecin & Block copolymeric micelles & IV & Prolonged systemic exposure \\
\hline
\end{tabular}

Nanotechnology in medical diagnostics
Diagnostics are considered as a facilitator for the successful prevention and efficient treatment of diseases 
which play an unsurmountable role in medicine. Nanotechnology has medical applications as biosensors and in molecular imaging both in vivo as well as in vitro, with respect to improved resolution and sensitivity, thus enabling earlier diagnosis of diseases. 'Theranostics' will take a new turn with the recent developments in nanotechnology. Few examples of in vitro diagnostics are microfluidic, nanoarrays, fluorescent labelling, local probes high resolution imaging, plasmonic and optical technique. On the other hand, in vivo diagnostics detect any abnormality instantaneously and help to ascertain therapeutic strategies. Nanobarcodes, nanowires, nanopores, nanocapacitors, nanotubes and nanosensors are the few examples among the vast range of the available diagnostic techniques. In multiple sclerosis, superparamagnetic iron oxide nanoparticles (SPION) are used frequently whereas quantum dots, metallic and semiconductor nanoclusters are the sophisticated tools used for cancer cell detection.

\section{CONCLUSION}

Nanotechnology has revolutionized the field of pharmaceuticals having an enormous potential as a carrier for spatial and temporal delivery of the bioactives. It provides boundless scope in the field of treatment, diagnostics and prognostics. When conventional technologies may be reaching their limits, nanotechnology will open a new horizon by providing new ingenious technologies in view of revenue loss caused due to offpatent drugs. The advantages of nano drug delivery over the conventional modalities are targeted and controlled release of drugs, improved sensitivity and precision for imaging and therapy, co-delivery of more than one drug, minimized toxicity to non-target cells and overcoming resistance. Last two decades have experienced the evolution of the "Magic bullet" paradigm, conceptualized by Richard Feynman that can be exploited to programme the much awaited personalized medicine. Although nanotechnology has helped to overcome the limitations of conventional therapeutics undoubtedly, several important issues like interplay of physicochemical features, nano particle toxicity need to addressed before translation from preclinical to clinical development.

\section{ACKNOWLEDGEMENTS}

Authors would like to thank Dr. Cuckoo Aiyappa for her continuous support and valuable advice in writing this article.

\section{Funding: No funding sources \\ Conflict of interest: None declared \\ Ethical approval: Not required}

\section{REFERENCES}

1. Riehemann K, Schneider SW, Luger TA, Godin B, Ferrari M, Fuchs H. Nanomedicine-challenge and perspectives. Angewandte Chemie International Edition. 2009;48(5):872-97.

2. Sanchez F, Sobolev K. Nanotechnology in concrete-a review. Construction and building materials . 2010;24(11):2060-71.

3. Sagadevan S, Periasamy M. A review on role of nanostructures in drug delivery system. Reviews on Advanced Materials Science. 2014;36(2):112-7.

4. Shivani S, Poladi KK. Nanosponges-NovelEmerging Drug Delivery System: A Review. International Journal of Pharmaceutical Sciences and Research. 2015;6(2):529.

5. Suri SS, Fenniri H, Singh B. Nanotechnology-based drug delivery systems. Journal of occupational medicine and toxicology. 2007;2(1): 16.

6. Nikalje AP Nanotechnology and its Applications in Medicine. Medchem. 2015;5:81-9.

7. Melnik AV, Shagalina OV. History of Nanotechnology. Siberian Federal University; 2011.

8. Rangasamy M. Nano technology: A review. Journal of Applied Pharmaceutical Science. 2011;(02): 08-16.

9. Roco MC. The long view of nanotechnology development: The National Nanotechnology Initiative at 10 years. 2011;427-45.

10. Muthu MS, Leong DT, Mei L, Feng SS. Nanotheranostics-application and further development of nanomedicine strategies for advanced theranostics. Theranostics. 2014;4(6):660-77.

11. Bamrungsap S, Zhao Z, Chen T, Wang L, Li C, Fu T, et al. Nanotechnology in therapeutics: a focus on nanoparticles as a drug delivery system. Nanomedicine. 2012;7(8):1253-71.

12. Maravajhala V, Papishetty $S$, Bandlapalli S. Nanotechnology in development of drug delivery system. International Journal of Pharmaceutical Sciences and Research. 2012;3(1):84.

13. Strebhardt K, Ullrich A. Paul Ehrlich's magic bullet concept: 100 years of progress. Nature Reviews Cancer. 2008;8(6):473-80.

14. Piktel E, Niemirowicz $K$, Wątek $M$, Wollny $T$, Deptuła P, Bucki R. Recent insights in nanotechnology-based drugs and formulations designed for effective anti-cancer therapy. Journal of nanobiotechnology. 2016;14(1):39.

15. Danhier F, Feron O, Préat V. To exploit the tumor microenvironment: passive and active tumor targeting of nanocarriers for anti-cancer drug delivery. Journal of Controlled Release. 2010;148(2):135-46.

16. Bhargav E, Madhuri N. Targeted Drug delivery-A review. WJPPS. 2013;3(1):150-9.

17. Sanna V, Pala N, Sechi M. Targeted therapy using nanotechnology: focus on cancer. International journal of nanomedicine. 2014;9:467.

18. Abbasi E, Aval SF, Akbarzadeh A, Milani M, Nasrabadi HT, Joo SW, et al. Dendrimers: synthesis, applications, and properties. Nanoscale Research Letters. 2014 Dec 1;9(1):247.

19. Surendiran A, Sandhiya S, Pradhan SC, Adithan C. Novel applications of nanotechnology in medicine. Indian J Med Res. 2009;689-701. 
20. Masareddy RS, Joshi VG, Sutar RS, Yellanki SK. Nano drug delivery systems - a review. Int J Sci Res. 2011;2(2):203-16.

21. Sowinska M, Urbanczyk-Lipkowska Z. Advances in the chemistry of dendrimers. New Journal of Chemistry. 2014;38(6):2168-203.

22. Mora-Huertas CE, Fessi H, Elaissari A. Polymerbased nanocapsules for drug delivery. International journal of pharmaceutics. 2010;385(1):113-42.

23. Prajapati BG. Nanoparticles as platforms for targeted drug delivery system in cancer therapy. Internet $\mathrm{J}$ Nanotechnol. 2009;3(1):1-8.

24. Hamelers IH, de Kroon AI. Nanocapsules: a novel formulation technology for platinum-based anticancer drugs. 2007; 445-53.

25. Singh A, Garg G, Sharma PK. Nanospheres: a novel approach for targeted drug delivery system. International Journal of Pharmaceutical Sciences Review and Research. 2010;5(3):84-8.

26. Suffredini G, East JE, Levy LM. New applications of nanotechnology for neuroimaging. American Journal of Neuroradiology. Am J Neuroradiol. 2014;35(7):1246-53.

27. Akbarzadeh A, Samiei M, Davaran S. Magnetic nanoparticles: preparation, physical properties, and applications in biomedicine. Nanoscale research letters. 2012;7(1):144.

28. Bañobre-López M, Teijeiro A, Rivas J. Magnetic nanoparticle-based hyperthermia for cancer treatment. Reports of Practical Oncology \& Radiotherapy. 2013;18(6):397-400.

29. Yadollahpour A, Rashidi S. Magnetic Nanoparticles: A Review of Chemical and Physical Characteristics Important in Medical Applications. Oriental Journal of Chemistry. 2015;31(1):25-30.

30. Smith L, Kuncic Z, Ostrikov K, Kumar S. Nanoparticles in cancer imaging and therapy. Journal of Nanomaterials. 2012;2012:10.

31. Maiti A, Bhattacharyya S. Review: Quantum dots and application in medical science. Int J Chem Chem Eng. 2013;3:37-42.

32. Srivastava NVK, Mishra U, Gupta T, Singh N. An overview on nanotechnology and nano-enabled drug delivery system. International Journal of Research in Pharmaceutical and Nano Sciences. 2015;4(5):33041.
33. Bentolila LA, Michalet X, Pinaud FF, Tsay JM, Doose $\mathrm{S}$, Li JJ, et al. Quantum dots for molecular imaging and cancer medicine. Discovery medicine. 2005;5(26):213.

34. Kraft JC, Freeling JP, Wang Z, Ho RJ. Emerging research and clinical development trends of liposome and lipid nanoparticle drug delivery systems. Journal of pharmaceutical sciences. 2014;103(1):29-52.

35. Sercombe L, Veerati T, Moheimani F, Wu SY, Sood AK, Hua S. Advances and challenges of liposome assisted drug delivery. Frontiers in pharmacology. 2015;6:286.

36. Chakraborty M, Jain S, Rani V. Nanotechnology: emerging tool for diagnostics and therapeutics. Applied biochemistry and biotechnology. 2011;165(5-6):1178-87.

37. Kulkarni PR, Yadav JD, Vaidya KA. Liposomes: a novel drug delivery system. Int J Curr Pharm Res. 2011;3(2):10-8.

38. Lin Z, Liu Y, MaX, Hu S, Zhang J, Wu Q, Ye W, Zhu S, Yang D, Qu D, Jiang J. Photothermal ablation of bone metastasis of breast cancer using PEGylated multi-walled carbon nanotubes. Scientific reports. 2015;5:11709.

39. Abdalla S, Al-Marzouki F, Al-Ghamdi AA, AbdelDaiem A. Different technical applications of carbon nanotubes. Nanoscale research letters. 2015;10(1):358.

40. He H, Pham-Huy LA, Dramou P, Xiao D, Zuo P, Pham-Huy C. Carbon nanotubes: applications in pharmacy and medicine. BioMed research international. 2013;2013.

41. Pilehvar S, De Wael K. Recent Advances in Electrochemical Biosensors Based on Fullerene-C60 Nano-Structured Platforms. Biosensors. 2015;5(4):712-35.

42. Reilly RM. Carbon nanotubes: potential benefits and risks of nanotechnology in nuclear medicine. Journal of Nuclear Medicine. 2007;48(7):1039-42.

43. Ramteke KH, Jadhav VB, Dhole SN. Microspheres: As carrieres used for novel drug delivery system. IOSRPHR. 2012;2(4):44-8.

Cite this article as: Das D, Maity N, Anuradha HV. Nanotechnology: a revolution in targeted drug delivery. Int J Basic Clin Pharmacol 2017;6:2766-73. 